\title{
Numerical Modeling of Shape and Topology Optimisation of a Piezoelectric Cantilever Beam in an Energy-Harvesting Sensor
}

\author{
Chung Ket Thein ${ }^{1 *}$, Jing-Sheng Liu $^{2}$ \\ ${ }^{1 *}$ School of Engineering and Physical Sciences, Heriot Watt University Malaysia, \\ No. 1, Jalan Venna P5/2, Precinct 5, 62200 Putrajaya, Malaysia \\ ${ }^{2}$ Department of Engineering, University of Hull, HU6 7RX, Hull, UK
}

\begin{abstract}
Piezoelectric materials are excellent transducers for converting mechanical energy from the environment for use as electrical energy. The conversion of mechanical energy to electrical energy is a key component in the development of self-powered devices, especially enabling technology for wireless sensor networks. This paper proposes an alternative method for predicting the power output of a bimorph cantilever beam using a finite element method for both static and dynamic frequency analyses. A novel approach is presented for optimising the cantilever beam, by which the power density is maximised and the structural volume is minimised simultaneously. A two-stage optimisation is performed, i.e., a shape optimisation and then a "topology” hole opening optimisation.
\end{abstract}

Key Words: piezoelectric; multi-disciplinary optimisation; shape and topology optimisation; energy harvesting; bimorph cantilever beam

\section{Introduction}

Rapid advances in electronic technology related to wireless sensor networks has resulted in a large demand for low-cost, maintenance-free, and self-powered wireless sensor nodes. There are many potential power sources for wireless sensor nodes, especially ambient vibrations around the node [1, 2]. It is possible to convert some of the ambient energy around the node into electrical energy using various methods, including the use of piezoelectric beams. In the context of energy harvesting, the development of renewable energy is important for protecting the environment. In advanced electronic technology, major reductions in the size and power consumption of CMOS (complementary metal-oxide semiconductor) circuit boards have made it easier to locate wireless sensors in inaccessible locations or hazardous environments.

The great potential of piezoelectric materials has stimulated numerous research efforts in this field. For example, Roundy used a rectangular piezoelectric cantilever beam to generate electrical energy from vibrational energy [3], and Miller et al. showed an increase in the weighted strain of a cantilever with the addition of a slit through the middle of the beam, yielding a weighted strain that is more than twice that of a rectangular cantilever [4]. Hence, the authors concluded that a typical solid rectangular cantilever beam is non-optimized for a micro-scale energy-scavenging generator. Roundy reported that the power density of a beam could be increased by using a smaller volume [5]. The author found that strain is distributed more evenly in the case of a trapezoidal cantilever beam, which generates more than twice the energy of a rectangular beam for a given volume. Several researchers have focused on maximising the power density of a piezoelectric generator. Mateu 
and Moll performed an analytical comparison between rectangular and triangular cantilevers in which they assumed uniform stress across the width of the cantilever [6]. Their analysis revealed that a triangular cantilever with the same beam volume as a rectangular beam has a higher average strain and maximum deflection for a given load, thereby producing more power per unit volume. Similar studies have been conducted by researchers $[7,8]$.

The power output of a piezoelectric generator is generally determined from the results of experiments. Roundy developed a general equation for calculating the power output of a rectangular beam [3]. This has subsequently led to a new approach to predicting the power output in which finite element method (FEM) has been used to analyse the behaviour of piezoelectric material [9]. However, few researchers have used FEM in optimising the best "topology" design to predict the power output/density of a piezoelectric cantilever beam.

The power output of a cantilever beam is directly related to the shape. This gives rise to the question of whether the topology of a trapezoidal cantilever beam is the best design in order to generate the maximum power density. To answer this question, the sensitivity of power density to beam shape is analysed in this research. In addition, hole openings within the cantilever beam are considered in order to seek an optimum "topology" structure which maximises the power density by using the minimum structural volume. In this research, FEM is also incorporated into the optimisation to simulate the behaviour of a cantilever beam, with the aim of simultaneously increasing the power output/power density. This background demonstrates the need to optimise the geometrical shape of a cantilever beam to yield the maximum power density.

A multi-objective and multi-discipline optimisation method (multifactor optimisation of structures technique, MOST), as originally developed by Liu and Hollaway [10] and Liu and Lu [11] to solve strength and stiffness challenges [12] and now extended to automatically accommodate and execute problems related to energy-harvesting optimisation. The MOST technique utilizes commercially available finite element codes (e.g., ANSYS) and combines finite element static analysis, dynamics analysis (for vibration frequency), and a unique optimisation technique, with the aim of simultaneously increasing both the power output and the power density. The MOST optimisation system can efficiently and systematically solve complex engineering-design problems, which may have multiple objectives and multiple disciplines, by performing a parameter profile analysis [13], thereby seeking the optimum solution. This method incorporates an assessment system which brings the scores and merit indices into a defined range (in this case 0-10) for all performance and loading cases. These features make MOST a powerful, cost-effective, and reliable tool with which to optimise complex structural systems.

This paper proposes an alternative method of predicting the power output/power density of a piezoelectric cantilever beam by static and dynamic (modal) analyses using the finite element method. A comparison between manual and automatic optimisation is discussed. A new method is presented of optimising a bimorph piezoelectric cantilever beam in an energy-harvesting application, with the aim of simultaneously maximising power output/power density and minimising structural volume, while also satisfying the strength and stiffness requirements of the structure. A two-stage optimisation is performed: firstly a shape optimisation and then a “topology” structural and power optimisation. 


\section{Predicting the power output/power density of piezoelectric generator designs with different geometrical} shapes/topology structures using finite element analysis

The material behaviour of sensors and actuators made of piezoelectric material can be modelled by the following constitutive equation [14]:

$$
\begin{aligned}
& \delta=\sigma / Y+d E \\
& D=\varepsilon E+d \sigma
\end{aligned}
$$

where $\delta$ is mechanical strain, $\sigma$ is mechanical stress, $Y$ is the modulus of elasticity, $d$ is the piezoelectric strain coefficient, $E$ is the electric field, $D$ is electric displacement, and $\varepsilon$ is the dielectric constant of the piezoelectric material.

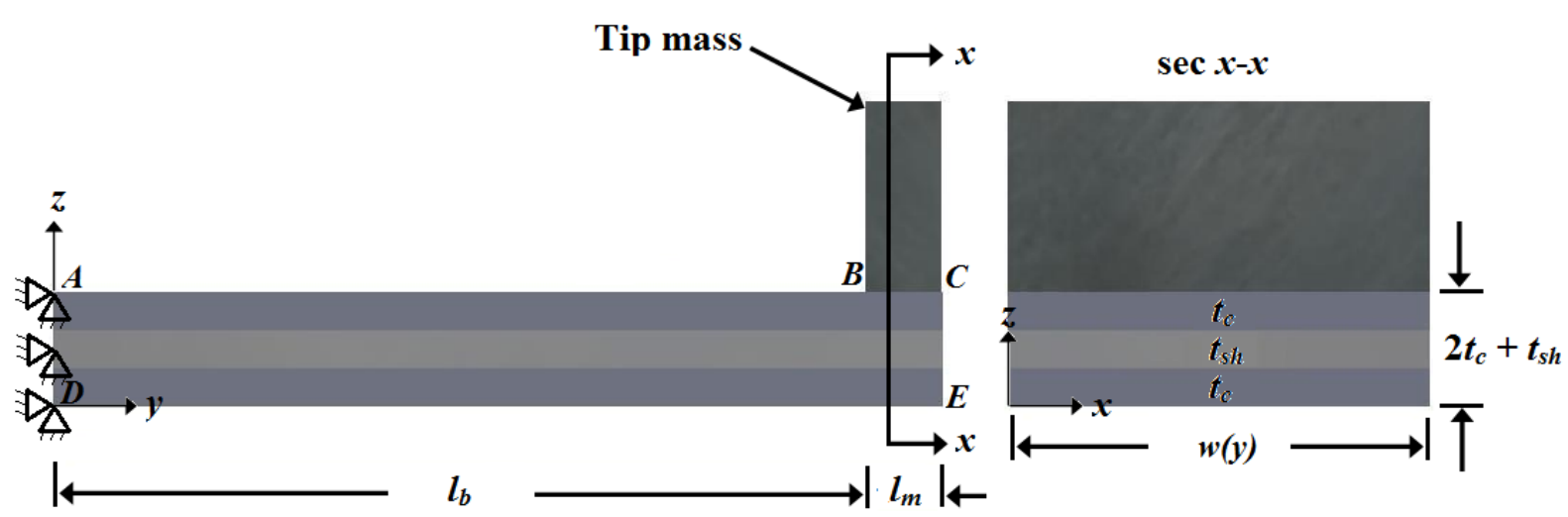

Fig. 1.Details of the piezoelectric cantilever beam

By using an analytical calculation, the effective moment of inertia $(I)$ of the cantilever beam is defined as follows:

$$
I=\left[\frac{2 w t_{c}^{3}}{12}+\frac{1}{2} w t_{c}\left(t_{c}+t_{s h}\right)^{2}\right]+\left[\frac{w t_{s h}^{3}}{12}\left(\frac{Y_{c}}{Y_{s h}}\right)\right]
$$

where $w$ is the beam width, $t_{c}$ is the thickness of the piezoelectric material, $t_{s h}$ is the thickness of the centre shim material, $Y_{c}$ is the Young's modulus of the piezoelectric material, and $Y_{s h}$ is the Young's modulus of the centre shim material. This equation is valid in calculating the effective moment of inertia, based on the rectangular shape shown in Fig. 1. Fig. 2 shows a schematic of a piezoelectric bender in which $l_{b}$ is the length of the base, $l_{m}$ is the length of the tip mass, $z_{t}$ is the vertical deflection of the cantilever beam tip, and $w(y)$ is the width of the piezoelectric material in terms of the electrode length $\left(l_{e}\right)$. 


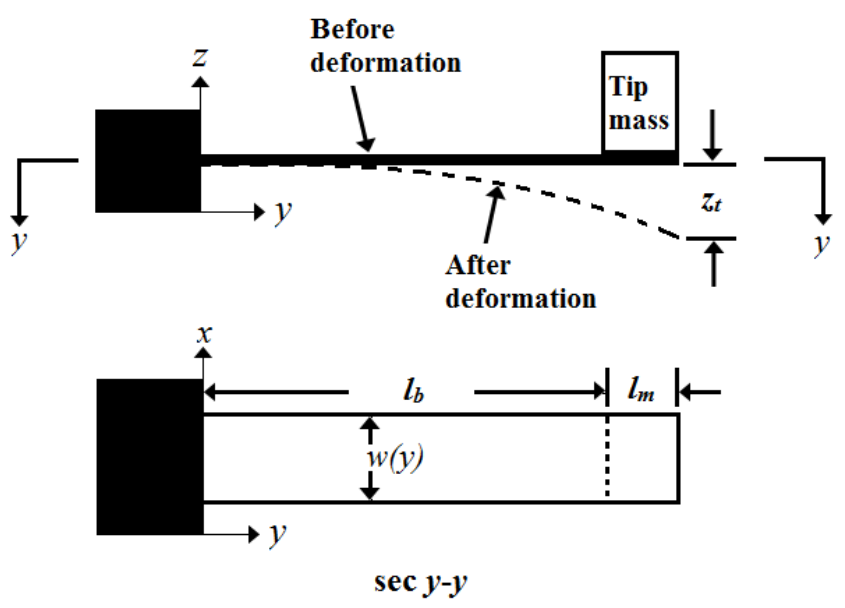

Fig. 2. Schematic piezoelectric bender

Fig. 1 shows a schematic diagram of the design domains, geometric constraints, load, and boundary conditions of the design. Points $A$ and $D$ are fixed at three coordinates $(x, y$, and $z$ ), and a concentrated pressure is applied at the free end of the cantilever beam (between $B$ and $C$ ). A tip mass is applied to the free end of the cantilever beam (known as a 'bimorph' system), which comprises three layers: the top and bottom layers of piezoelectric material, and a middle layer (a brass shim). The electrode, used to produce energy, is connected to the top and bottom surfaces. The electrode length ( $l_{e}$; not shown in Fig. 2$)$ is always equal to or less than the beam length $\left(l_{b}\right)$. When the beam is deformed, a stress is induced on the top and bottom surfaces. For the piezoelectric generator, stress and strain are the main concerns. The stress and strain in a piezoelectric material are the average element stress and strain, respectively. Therefore, the average element stress $\sigma_{\text {ave }}$ in the piezoelectric material covered by the electrode is as follows:

$$
\sigma_{\text {ave }}=\frac{1}{l_{e}} \int_{0}^{l_{e}} \frac{M(y) b}{I} d x
$$

where $M(y)$ is the moment in the beam as a function of the distance $(y)$ from the fixed end and $b$ is the distance from the centre of the shim to the centre of the piezoelectric layer. If the piezoelectric cantilever beam has a rectangular shape, the average element stress can be calculated using Eq. (4), as can the bending moment of the beam. However, if the beam has an irregular shape, it becomes difficult to calculate the second moment of the area. Finite element analysis can be used to obtain the solution of the average element stress for each analysis, meaning that the second moment of the area is not directly required to calculate the average element stress of the beam, especially in the case of an irregular shape. Consequently, the average element stress can be stated as follows:

$$
\sigma_{\text {ave }}=\frac{1}{n_{c}} \sum_{c=1}^{n_{c}} \sigma_{c}
$$

where $n_{c}$ is the total number of piezoelectric material elements. This approach assumes that all the elements are of equal size. Roundy (2003) stated that the tip deflection of the cantilever beam is related to the average strain in the piezoelectric material. By utilising Hooke's Law for elastic material, the average element stress from 
finite element analysis, and the Roundy method (Roundy, 2003), the relationship among these factors can be defined as follows:

$$
b^{*}=\frac{\sigma_{a v e}}{Y_{c} z_{t}}
$$

Where $b^{*}$ is related to the element average stress, Young's modulus of the piezoelectric material and vertical deflection at the free end.

The mechanical damping ratio $(\zeta)$ of the system can be stated as follows:

$$
\zeta=\frac{c}{2 m_{e f f} \omega_{n}}
$$

where $m_{\text {eff }}$ is the effective mass, $\omega_{n}$ is the natural frequency of the system, and $c$ is a damping coefficient. The effective mass and natural frequency can be found from the finite element analysis. Next, the capacitance of the beam is defined as

$$
C_{p}=\int_{0}^{l_{e}} \frac{n_{p c} \varepsilon w(y)}{t_{c}} d x
$$

where $n_{p c}$ is the number of piezoelectric layers and $\varepsilon$ is the dielectric constant of the piezoelectric material. The power transferred to the load is simply square of the voltage divided by load resistance, $V^{2} / R$. By using the solution of the average element stress and the vertical tip displacement, the power output $(P)$ of the beam (vibrating at its resonance frequency) is then formulated as follows:

$$
P=\frac{1}{2 \omega_{n}^{2}} \frac{R C_{p}^{2}\left(\frac{d t_{c} \sigma_{a v e}}{\varepsilon Z_{t}}\right)^{2}}{\left(4 \zeta^{2}+k^{4}\right)\left(R C_{p} \omega_{n}\right)^{2}+4 \zeta k^{2}\left(R C_{p} \omega_{n}\right)+(2 \zeta)^{2}} A_{i n}^{2}
$$

where $R$ is the load resistance, $d$ is the piezoelectric strain coefficient, $k$ is a coupling coefficient, and $A_{\text {in }}$ is the magnitude of the input acceleration. Eq. (9) was verified with the theoretical calculations and yielded a percentage error of $4.29 \%$ [15]. The optimum resistance can be found by differentiating Eq. (9) with respect to $R$, setting the result equal to zero and solving for $R$. The optimum resistance $\left(R_{o}\right)$ is as follows:

$$
R_{o}=\frac{2 \zeta}{\omega_{n} C_{p} \sqrt{4 \zeta^{2}+k^{4}}}
$$

The power density $\left(P_{d}\right)$ per unit volume of piezoelectric material is defined as

$$
P_{d}=\frac{P \cdot \rho_{c}}{\int_{0}^{l_{e}} n_{c} t_{c} w(y) d x}
$$

where $\rho_{c}$ is the piezoelectric material density. 


\section{Investigation of the "topology" structure of the cantilever beam}

To increase the strain energy, additional holes within the bimorph cantilever beam are considered. Miller et al. [4] removed some underlying silicon (which is used to glue layers of material) by releasing gaseous etch and obtained a strain increase of at least $30 \%$. In this research, "topology" hole openings within a cantilever beam is investigated to optimise the output power density. The aim of the investigation is to prove that the power density of the topologically holed structure is better than that of an unholed structure. Additional holes were applied to the region of the cantilever beam until the maximum output power was attained.

A cantilever beam is modelled and analysed using ANSYS finite element software. The ANSYS SOLID92 element is used to generate the model rather than the SOLID98 element, although both elements are 10-node tetrahedral shapes with a large deflection and stress-stiffening behaviour. The SOLID92 element adapts well to the free meshing of irregular shapes. Both static analysis and dynamic (modal) analysis (for vibration frequency) are performed. The initial model consists of 9620 elements (both piezoelectric and shim elements) with a uniform element length of $0.8 \mathrm{~mm}$. The initial dimensions of the beam are listed in Table 1 (see Fig. 1 for parameter details). The values of the mechanical and electric properties of the piezoelectric material (PZT5A4E) and brass shim are given in Table 2 [16, 17].

Table 1 Dimensions of the initial design

\begin{tabular}{lc}
\hline Parameters & Initial value $(\mathrm{mm})$ \\
\hline Thickness of piezoelectric material $\left(t_{c}\right)$ & 0.19 \\
Thickness of shim material $\left(t_{s h}\right)$ & 0.13 \\
Length of base $\left(l_{b}\right)$ & 21.5 \\
Length of tip mass $\left(l_{m}\right)$ & 2.00 \\
Effective length of piezoelectric material PZT $\left(l_{e}=l_{m}+l_{b}\right)$ & 23.5 \\
Width $(w(y))$ & 12.7 \\
\hline
\end{tabular}

Table 2 Mechanical and electrical properties employed in the finite element analysis

\begin{tabular}{lcc}
\hline Material properties & $\begin{array}{c}\text { Piezoelectric } \\
\text { material }\end{array}$ & Brass shim \\
\hline Young modulus $(\mathrm{GPa})$ & 66 & 117 \\
Yield stress $(\mathrm{MPa})$ & $24^{*}$ & 200 \\
Maximum deflection $(\mu \mathrm{m})$ & 300 & - \\
Poisson's ratio & 0.31 & 0.324 \\
Density $\left(\mathrm{kg} / \mathrm{m}^{3}\right)$ & 7800 & 7165 \\
Relative dielectric constant & 1800 & - \\
$\mathrm{d} 31(\mathrm{~m} / \mathrm{V})$ & $-190 \times 10^{-12}$ & - \\
\hline
\end{tabular}

*Dynamic peak tensile strength [18] 


\subsection{Uni-hole topology "search"}

The aim of the optimisation performed in this research is to maximise the power density, and to minimise the structural volume. In this example, holes with a constant size are added and shifted to seek the optimum "topology" of a bimorph cantilever beam. The focus here is on the effect of the hole location on the power output with the same volume constraint. The initial hole diameter is just randomly selected and the hole size is not part of the investigation in this example. . This is done by using a manual procedure to seek the maximum power density. All the ANSYS simulation results show a constant volume except design 1, which is an unholed structure (see Fig. 2). Designs 2-4 are a uni-hole topology with variable distance from the free end of the cantilever beam and a constant diameter of $4.4 \mathrm{~mm}$. Fig. 3 shows the details of the designs.

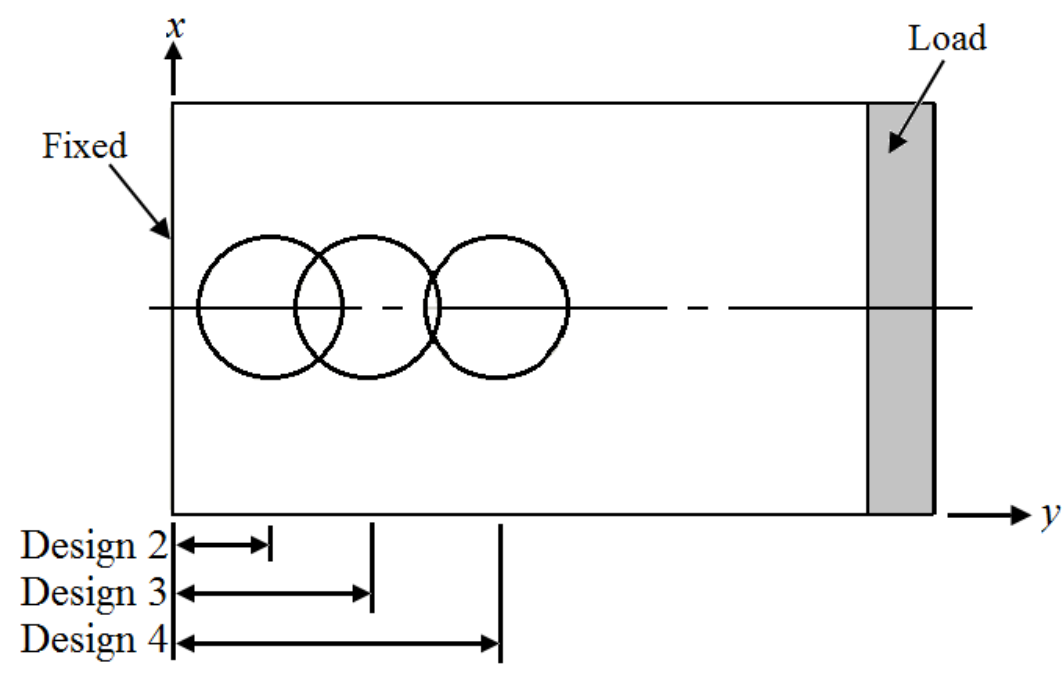

Fig. 3. Load, boundary conditions, and hole location in each design

Table 3 shows the attributes of the designs and compares the power density. The power density of design 1 is $1.99 \mu \mathrm{W}$, which is greater than that for designs 2 and 3. This result reflects the fact that the capacitance of the beam and the average stain energy per unit volume is higher for design 1 than for designs 2 and 3. In addition, the holed structures in designs 2 and 3 have removed an area of higher average stress. However, when the hole is located near the free end of the cantilever beam, the power density is doubled that for design 3 , indicating that the strain energy at the free end of the beam is smaller than that at the fixed end.

Table 3 Power density of uni-hole

\begin{tabular}{ccc}
\hline Design & $\begin{array}{c}\text { Power density } \\
(\mu \mathrm{W})\end{array}$ & Differences (\%) \\
\hline 1 & 1.99 & - \\
2 & 1.94 & -2.142 \\
3 & 1.95 & -1.955 \\
4 & 2.06 & 3.624 \\
\hline
\end{tabular}


Fig. 4 compares several sets of power density data against the distance (constant hole size) along the centre line of the beam. This represents a manual search method designed to obtain the maximum power density. From Fig. 4, the maximum power density is approximately $2.18 \mu \mathrm{W}$ at a distance of about $17 \mathrm{~mm}$ from the fixed end. This method may be viable in solving a single variable with a simple design. If the design involves multiple design variables, objectives, and constraints, it is difficult to achieve an optimum solution by using a manual search method. In such cases, a fully automatic optimisation should be used, as described in the Section 4.

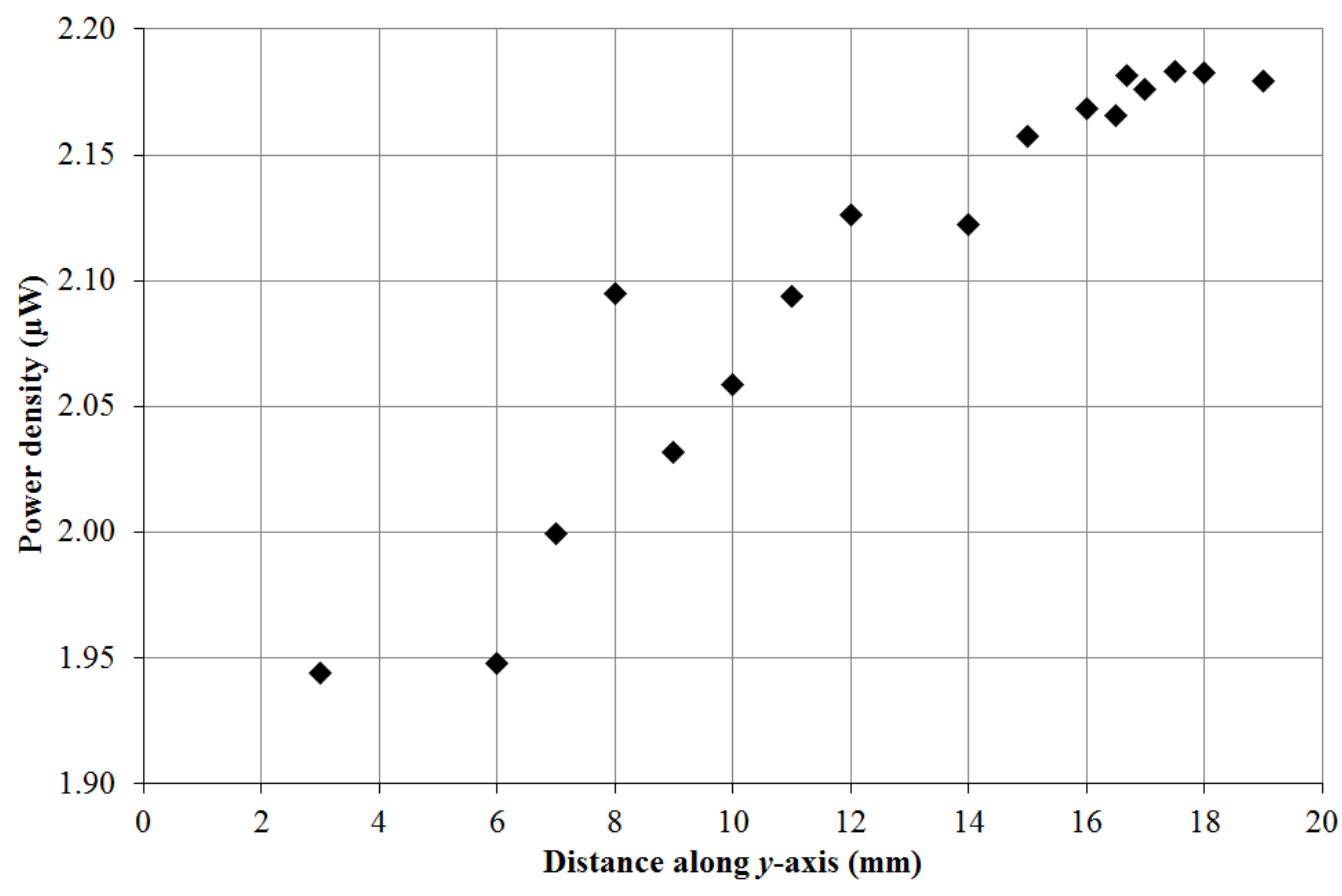

Fig. 4. Power density obtained for different hole locations in a rectangular bimorph cantilever beam

\section{Electrical energy and structural optimisation of a bimorph piezoelectric cantilever beam}

The power output of a cantilever beam is directly related to its shape and structure. This study considers the sensitivity of the power to the shape of the cantilever. The main objective is to find the optimum geometrical shape of a bimorph cantilever beam that yields the maximum power and has the minimum structural volume using a fully automatic optimisation technique. In addition, the beam must satisfy the strength and stiffness requirements. A dynamic analysis (for the vibration frequency) is also required in the power calculation, as indicated in Eq. (9).

\subsection{Formulation of the optimisation problem}

As part of this study, the optimisation method MOST is used in conjunction with the ANSYS finite element software. The design problem is therefore to maximise the power density and simultaneously minimise the structural volume, subject to the strength and stiffness constraints. The optimisation to be solved is stated as follows:

$$
X=\left(x_{1}, x_{2}, \ldots, x_{k}\right)
$$




$$
\begin{array}{ll}
\min & \left\{V_{s}(X)\right\} \\
\text { and } & \\
\max & \left\{P_{d_{, j}}(X)\right\} \\
\text { s.t. } & \left\{P_{d, j} \geq P_{d, \text { ini,j }} ; V_{s} \leq V_{s, \text { ini }} ; \sigma_{\text {ave }, j} \geq \sigma_{\text {ini }, j} ; \sigma_{\text {max }, j} \leq \sigma_{\mathrm{y}} ; \delta_{\text {ini }, j} \leq \delta_{j} \leq \delta_{\text {lim }, j}\right\} \text { and } \\
& \left\{x_{i}^{\min } \leq x_{i} \leq x_{i}^{\max }, i=1,2, \ldots, k\right\} \\
& j=1,2, \ldots, n
\end{array}
$$

where $k$ is the number of design variables, $V_{s}$ is the structural volume (excluding the volume of the tip mass), $\sigma_{\text {ave }}$ is the average element stress of the structure, $\delta$ is the displacement of point $E$ (see Fig. 1 ), $\sigma_{\max }$ is the maximum von Mises stress of the structure, and $P_{d}$ is the power density. The subscript 'ini' indicates the initial value for the structure (here, the initial iteration when $n_{i}=0$ ) and $n$ is the number of loading cases (here, $n=1$ ). The subscript 'lim' indicates a specified performance limit for the structure. In this research, the cantilever beam is optimised to carry a tip mass of $4.1 \mathrm{~g}$ with a maximum vertical displacement of $\delta_{\text {lim }}=300 \mu \mathrm{m}$ at any node, satisfying a maximum strength of $\sigma_{y}=24 \mathrm{MPa}$ (see Table 2). $x_{i}^{\min }$ and $x_{i}^{\max }$ are the lower and upper bounds of the design variables of $x_{i}$, respectively. In this case, the design variables are the widths of the beam, the geometrical sizes of the hole openings, and/or the locations of the "topology" shapes of the cantilever beam.

\subsection{Optimisation methodology}

The requirements for a complex structural design dictate that the optimisation must involve multiple objectives, multiple loading cases, and a large number of design variables. Thus, an important part of the optimisation procedure is to establish a suitable method for evaluating this process; however, complex crossrelationships make it difficult to suitably appraise the design in order to yield an overall quantitative performance index which truly represents the character of the system. MOST tackles this problem by employing a systematic method for evaluation based on the concept of parameter profiles analysis [13]. This method evaluates a structural design by considering many individual performance parameters for a variety of loading cases, while also considering mass.

An $m \times n$ matrix $\left(d_{i j}\right)$ - the so-called performance data matrix (PDM) —is defined by a set of performance parameters $P_{i}(i=1,2, \ldots, m)$ and loading case parameters $C_{j}(j=1,2, \ldots, n)$. The PDM is a schematic representation of a collection of data, as shown in Table 4 . Thus, the data point $d_{i j}$ is the $i$-th performance $P_{i}$ of the structure for loading case $C_{j}$. In this case, the data points of the matrix are obtained by a finite element analysis of the structure. The matrix lists every item of the loading cases as well as every performance parameter relevant to the individual loading cases. 
Table 4 Performance data matrix

\begin{tabular}{ccccc}
\hline & $C_{1}$ & $C_{2}$ & $\cdots$ & $C_{n}$ \\
\hline$P_{1}$ & $d_{11}$ & $d_{12}$ & $\cdots$ & $d_{1 n}$ \\
$P_{2}$ & $d_{21}$ & $d_{22}$ & $\cdots$ & $d_{2 n}$ \\
$\vdots$ & $\vdots$ & $\vdots$ & & $\vdots$ \\
$P_{m}$ & $d_{m 1}$ & $d_{m 2}$ & $\cdots$ & $d_{m n}$ \\
\hline
\end{tabular}

A parameter profile matrix (PPM) is created to review the profile of the performances for different loading cases (Table 5). To simplify the calculations, the values of the performance indices are normalised to the range $0-10$. This enables different loading cases and parameters to be compared, in order to gain an overall perspective of the characteristics of the system. The PPM assesses the character of the structure with respect to the actual performances at their worst acceptable limits and the best expected values of the performances.

Table 5 Parameter profile matrix

\begin{tabular}{ccccc}
\hline & $C_{1}$ & $C_{2}$ & $\cdots$ & $C_{n}$ \\
\hline$P_{1}$ & $D_{11}$ & $D_{12}$ & $\cdots$ & $D_{1 n}$ \\
$P_{2}$ & $D_{21}$ & $D_{22}$ & $\cdots$ & $D_{2 n}$ \\
$\vdots$ & $\vdots$ & $\vdots$ & & $\vdots$ \\
$P_{m}$ & $D_{m 1}$ & $D_{m 2}$ & $\cdots$ & $D_{m n}$ \\
\hline
\end{tabular}

The data point $\mathrm{D}_{i j}$ for one acceptable limit (e.g., the lower limit) is calculated as follows:

$$
D_{i j}=\frac{d_{i j}-l_{i j}}{b_{i j}-l_{i j}} \times 10
$$

where $d_{i j}$ is the actual value of the performance obtained from the PDM, and $l_{i j}$ and $b_{i j}$ are the lower acceptable limit and the best expected value, respectively. Eq. (12) is valid for $l_{i j}<d_{i j}<b_{i j}$; for $d_{i j}>b_{i j}, D_{i j}=10$; and for $d_{i j}<l_{i j}, D_{i j}=0$. The data point for the cases of the acceptable upper limit and double acceptable limits can be calculated in a similar way.

The mean and standard deviation (SD) are calculated for each parameter and loading case in each column and row in the PPM. A well-designed system should have low SDs and high mean values (close to 10). The existence of high SDs signifies that the system is likely to have significant problematic areas. Therefore, a high SD for a row indicates variable system performance at different loading cases for a particular parameter. Conversely, a high SD for a column indicates the system is likely to have significant problematic performance for the specific loading case.

The system can be further analysed using a parameter performance index (PPI) and a case performance index (CPI), which are defined as follows: 


$$
P P I_{i}=\frac{n}{\sum_{j=1}^{n} 1 / D_{i j}} \quad, \quad i=1,2, \cdots, m \text { and } C P I_{j}=\frac{m}{\sum_{i=1}^{m} 1 / D_{i j}} \quad, \quad j=1,2, \cdots, n
$$

When thei-th parameter is very vulnerable, some data points $D_{i j}$ of the PPM will have values close to 0 and hence the $\mathrm{PPI}_{i}$ will also be close to 0 . Similarly, when the system is vulnerable at the $j$-th loading case, $\mathrm{CPI}_{j}$ will be close to 0 . The highest values for PPI and CPI are 10. PPI and CPI values close to 10 indicate good design, whereas values close to zero indicate poor design. The mean values, CPIs, PPIs, and SDs provide an overall performance assessment for the system and loading cases. These indices are calculated by summing the inverse of the data points as a performance rating to avoid the effect associated with low scores being hidden by high scores. The mean values are not used directly to rate the performance. The system may be reviewed by using the information in the indices, as follows:

- A comparison of PPIs indicates whether the system performs better with respect to some performances than to others.

- A comparison of CPIs shows whether the system performs better under certain loading cases than under others.

According to the matrix profile analysis, PPI and CPI are measures of the vulnerability of each performance parameter and each loading case, respectively. Hence, the integration of PPI and CPI indicates the vulnerability of a particular parameter/loading case combination. An overall performance index (OPI) is used to develop the overall objective function. The OPI, which takes the form of a qualitative score, can be established for the system by considering all the performances and all the loading cases. The OPI function lies in the range of 0 100. The OPI can be expressed as follows:

$$
O P I=\frac{100}{m \times n} \sum_{i=1}^{m} \sum_{j=1}^{n} W_{P_{i}} \cdot P P I_{i} \times W_{C_{j}} \cdot C P I_{j}
$$

where $W_{p_{i}}$ and $W_{c_{j}}$ are weighting factors (in the range of $0-1$ ) that reflect the preference for each performance parameter and each loading case. The OPI can be used to compare the performances of different designs: the higher the OPI score, the more reliable the design. The objective function is maximised using the effective zeroorder method, employing conjugate search directions [10]. An effective polynomial interpolation unidimensional search method is also used in the algorithm. This optimisation technique has the advantage of forcing the performances to approach their optimal values. The nearer the performances to the acceptable limits, the stricter the 'punishment' (penalties).

\section{Numerical model on shape and "topology" design for the bimorph cantilever beam}

In this example, the power density is maximised and the structural volume is simultaneously minimised using the MOST optimisation technique. A two-stage optimisation is performed. The first stage seeks the optimum shape of the cantilever beam, as well as the maximum output power. The second stage is concerned with design optimisation while maximising the power density and minimising the structural volume. 


\subsection{Stage 1 - Shape optimisation}

The design parameters of the first stage optimisation are shown in Fig 5. There are 9 geometrical design parameters (W1-W9) in which the original width is set to be $12.7 \mathrm{~mm}$. Each geometrical design parameters are bounded to a given range , (i.e., $0.5 \mathrm{~mm} \sim 6.4 \mathrm{~mm}$ ), and the structure is kept symmetric about the central line of the beam throughout the optimisation process. Each design variables are free to move within the given range in the $\mathrm{x}$-axis direction. Each point are then connected through a spline to create a smooth curve. In addition, a weighting system is applied to the parameters in the optimisation process based on the importance. In this paper, four performances need to be focused, i.e., the maximum von-Mises stress, the maximum displacement, the volume of the beam, and the power output. Each performance will be tied to one weighting factor. Various combinations of the weighting factors are feasible in the optimisation and it will produce different geometrical shapes.

\section{symmetry axis}

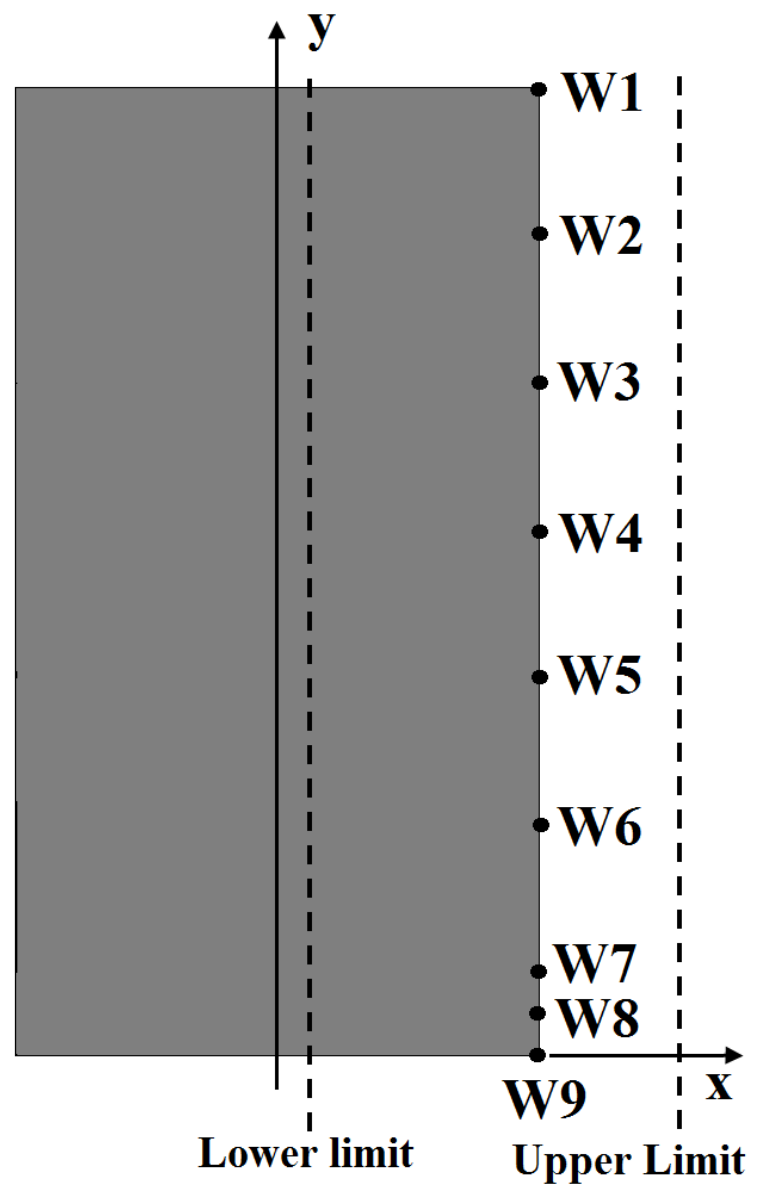

Fig. 5. Design variables of the initial design in Stage 1 
The load and boundary properties are shown in Fig. 1, and the mechanical and electrical properties are listed in Tables 1 and 2, respectively. The first stage focuses on maximising the output power by obtaining the optimal geometrical shape for an idealised bimorph cantilever beam. The optimisation is started from a rectangular cantilever beam. The optimisation took 27 iterations to converge, as shown in Fig. 6, which depict the evolution of the structural volume, the power output, and the power density. Fig. 7 shows the distribution of von Mises stress for the initial and optimised designs.

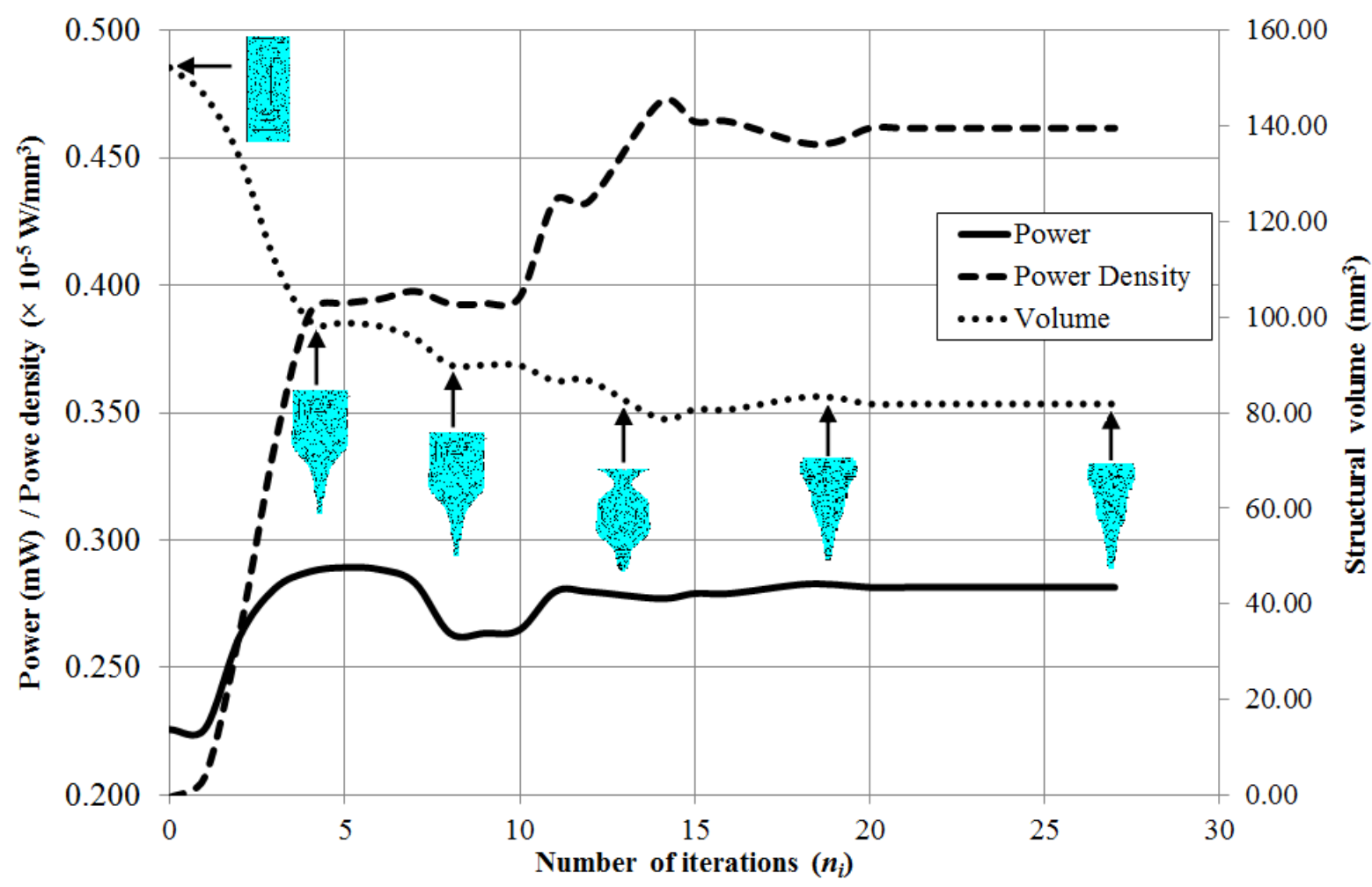

Fig. 6.Optimisation convergence history of the power, power density and structural volume

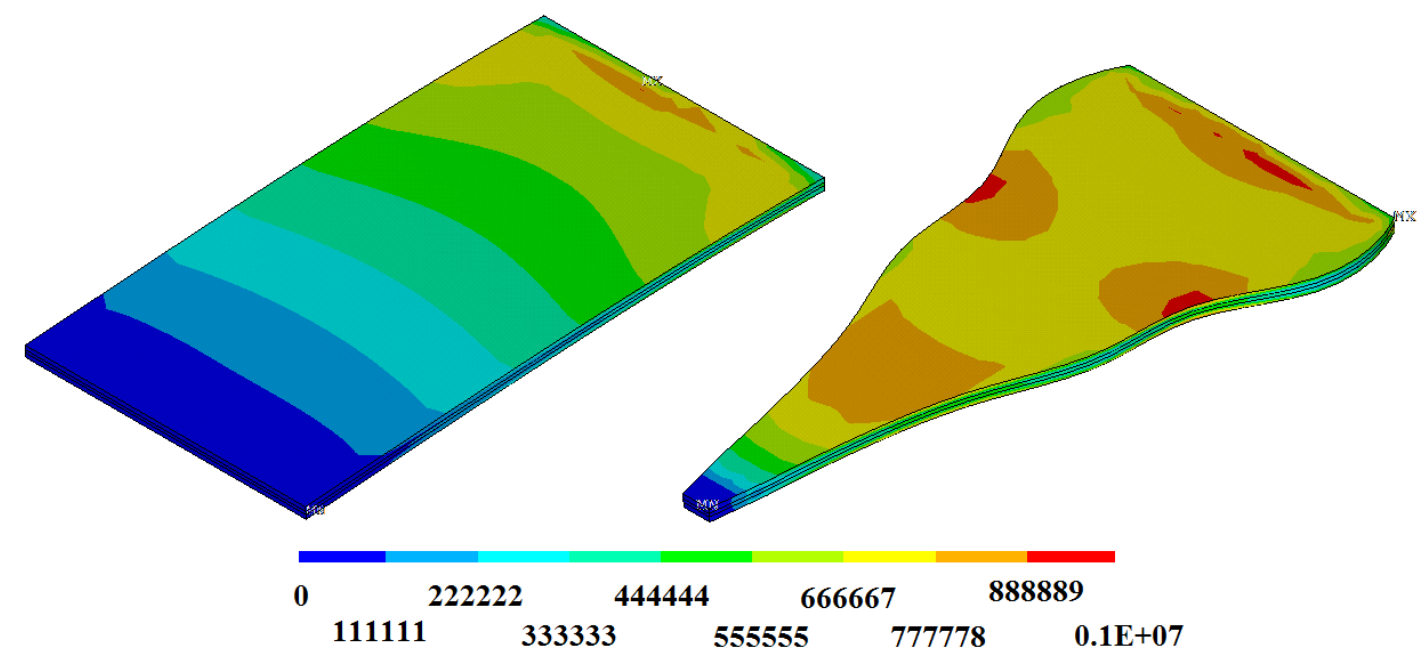

Fig. 7. Distribution of von Mises stress for the initial design (left) and for the optimised design (right) (Pa) 
The attributes of the initial and optimised designs are given in Table 6. The output power is increased from approximately 0.226 to $0.282 \mathrm{~mW}$, which corresponds to an increase of approximately $25 \%$ compared with the initial design (see Fig. 5). The structural volume (piezoelectric and shim material combined) is reduced significantly from 152.21 to $81.83 \mathrm{~mm}^{3}$, representing a $46.2 \%$ saving in materials. The power density of the optimised design is more than twice that of the initial design, and the vertical deflection of the cantilever beam is increased by about $47 \%$, from 8.48 to $12.50 \mu \mathrm{m}$. The maximum von Mises stress shows an increase from 0.89 to 1.00 MPa. These results are well within the stiffness and strength constraints $\left(\delta_{\lim }=300 \mu \mathrm{m}\right)$ and yield stress $\left(\sigma_{y}=24 \mathrm{MPa}\right)$.

Table 6 Design attributes of the initial and optimised designs of a cantilever beam

\begin{tabular}{lcc}
\hline & Initial design & $\begin{array}{c}\text { Optimised } \\
\text { design }\end{array}$ \\
\hline Power $(\mathrm{mW})$ & 0.226 & 0.282 \\
Volume of piezoelectric material $\left(\mathrm{mm}^{3}\right)$ & 113.41 & 60.97 \\
Volume of shim material $\left(\mathrm{mm}^{3}\right)$ & 38.80 & 20.86 \\
Power density $\left(\mu \mathrm{W} / \mathrm{mm}^{3}\right)^{*}$ & 1.99 & 4.62 \\
Maximum von Mises stress $(\mathrm{MPa})$ & 0.89 & 1.00 \\
Average element stress $(\mathrm{MPa})$ & 0.24 & 0.46 \\
Maximum vertical displacement $(\mu \mathrm{m})$ & 8.48 & 12.50 \\
Capacitance $(\mathrm{nF})$ & 50.07 & 26.92 \\
Frequency $(\mathrm{Hz})$ & 113 & 92 \\
\hline
\end{tabular}

*per unit volume of piezoelectric material

\subsection{Stage 2 - Shape and "Topology" optimisation}

The above initial investigation demonstrated the validity of the MOST-optimised structure for the cantilever beam. This result paves the way for the application of MOST in terms of shape optimisation, which is the first stage of optimisation.

The Stage 2 involved the design optimisation and discretisation of the shape and "topology" for hole openings of a structure, while satisfying the requirements regarding strength and power density. Additional hole openings were applied to the domain region of the first-stage optimised structure until the maximum power density was attained. This process was based on maximising the power output and minimising the structural volume. The trade-off between structural volume and output power of a structure represents a great challenge in design optimisation. The removal of material results in reduced structural volume, which may affect the maximum power output.

The schematic diagram in Fig. 8 shows the design domain of the initial structure (optimised in the first stage). The load and boundary conditions of the cantilever beam remain unchanged (see Fig. 1). All the elements within the outer boundary are kept 'frozen'. The removal of material occurs within the confines of the design domain. 


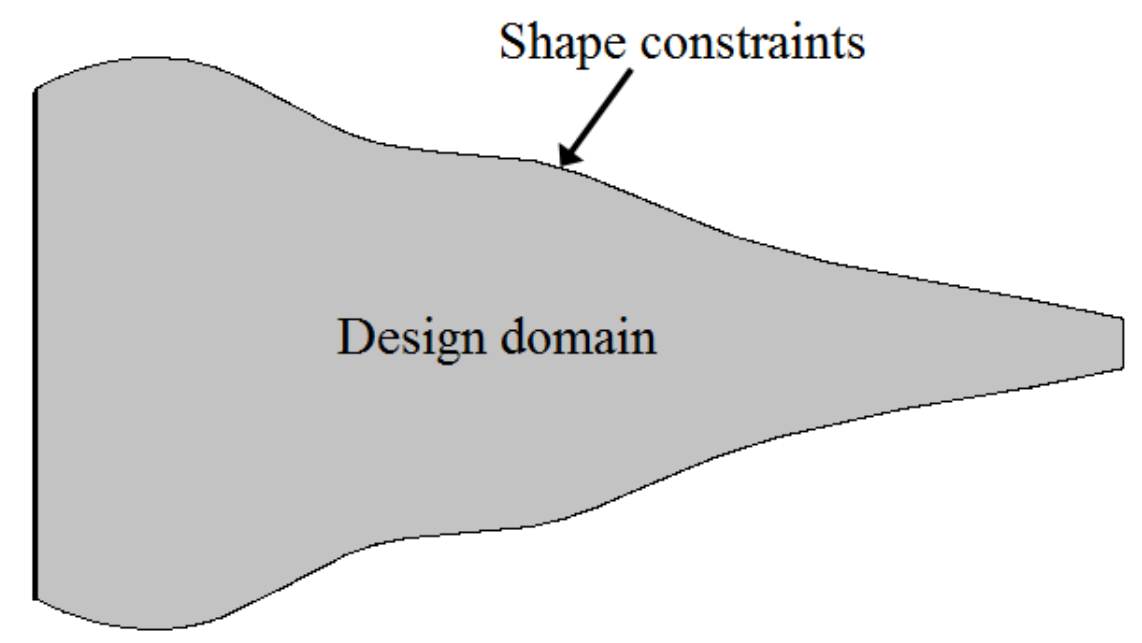

Fig. 8. Design domain and shape constraints of the optimised design

The optimised design (first stage) of the bimorph cantilever beam is modelled using ANSYS, as shown in Fig. 8. The bimorph cantilever beam with the first-stage optimised design is considered to obtain an improved design. Using the MOST technique, "topology" related optimisation could be performed subjected to hole openings as the initial setups in initiating the optimisation processes. In this paper, the initial design with random hole opening sizes of the "topology" shapes are shown in Fig. 9. The manufacturing capability is not considered during the optimisation procedure and the weighting factors are the same as in the Stage 1. 


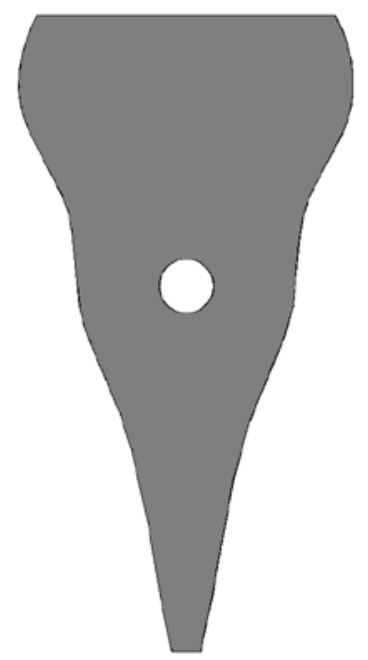

(a)

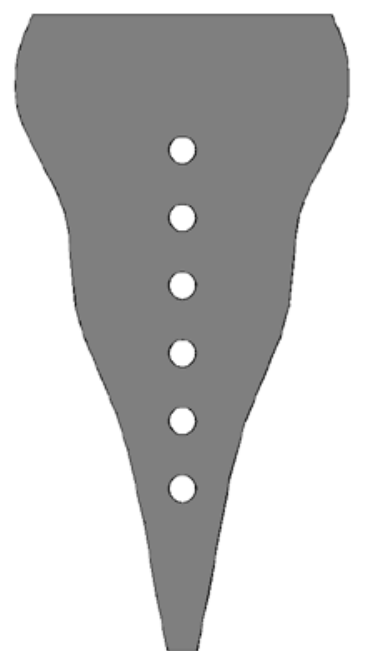

(e)

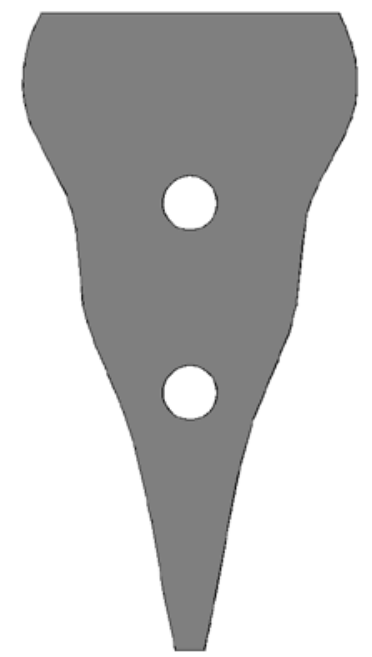

(b)

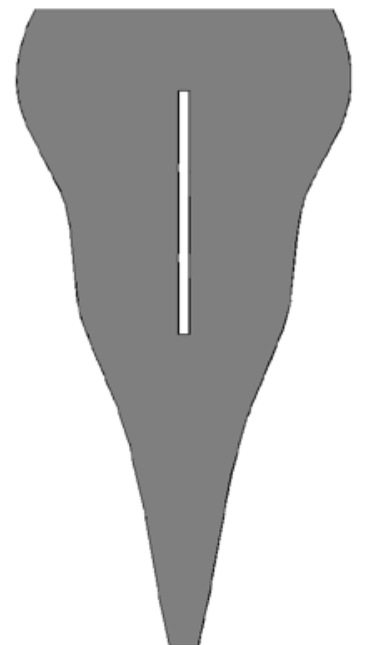

(f)

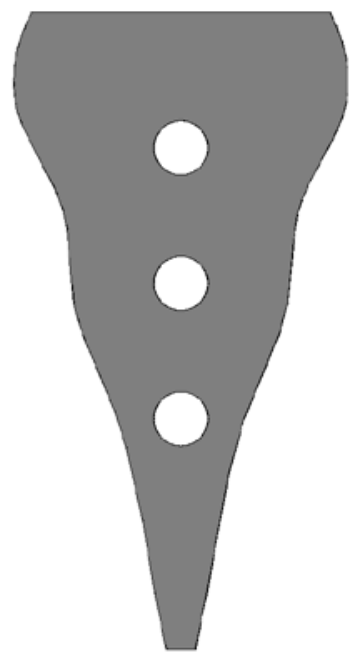

(c)

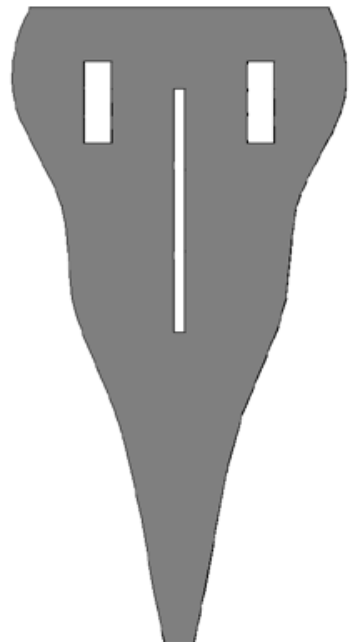

(g)

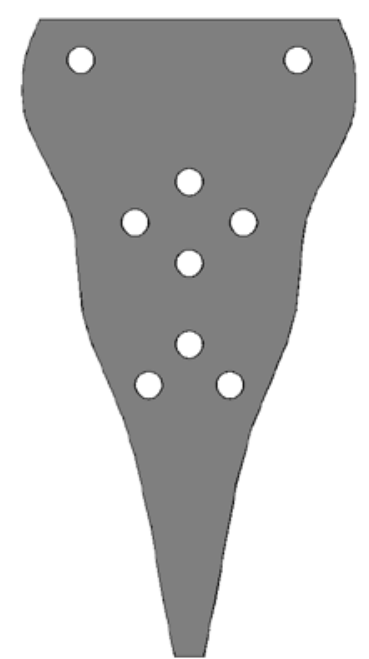

(d)

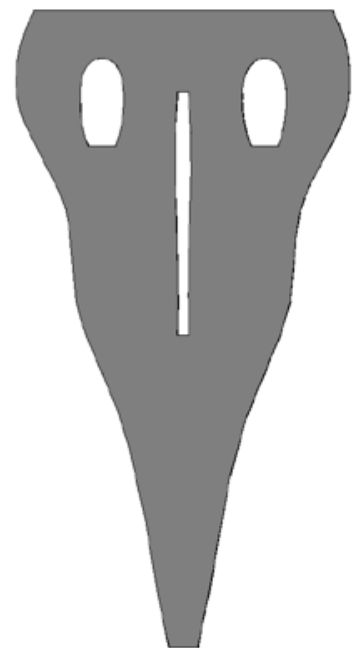

(h)

Fig. 9. Initial “topology” hole openings, showing designs (a) to (h)

The optimised designs are presented in Fig. 10 which shows that eight different designs with different numbers and sizes of holes in the design domain. Each design are sought by performing a fully automatic MOST optimisation technique in order to maximise the overall performance index (OPI). Designs (a) to (e) are focused on the various circular hole diameters and locations in seeking higher power outputs. In addition, designs (f) to (h) are focused on the different geometrical hole openings. 


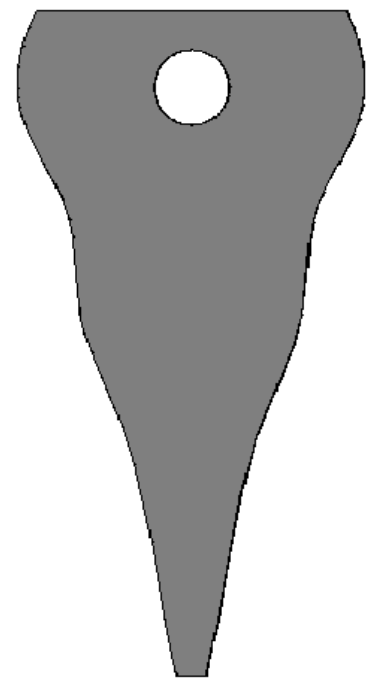

(a)

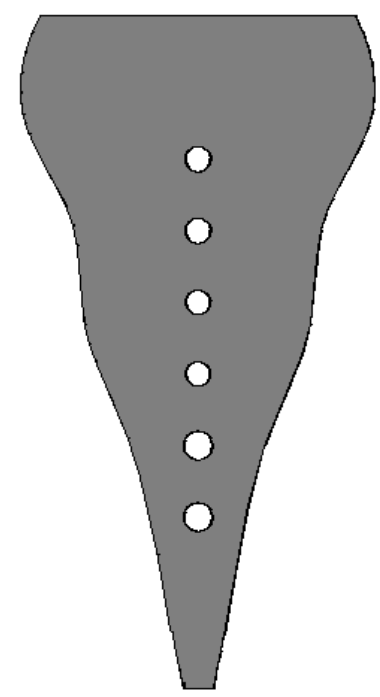

(e)

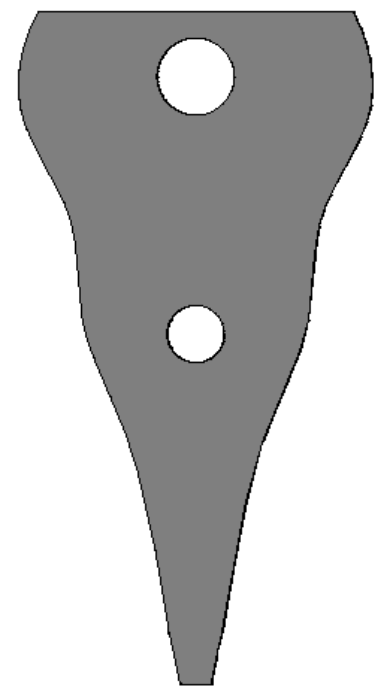

(b)

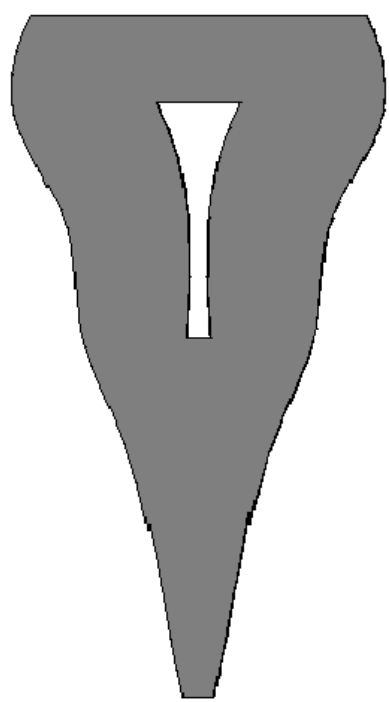

(f)

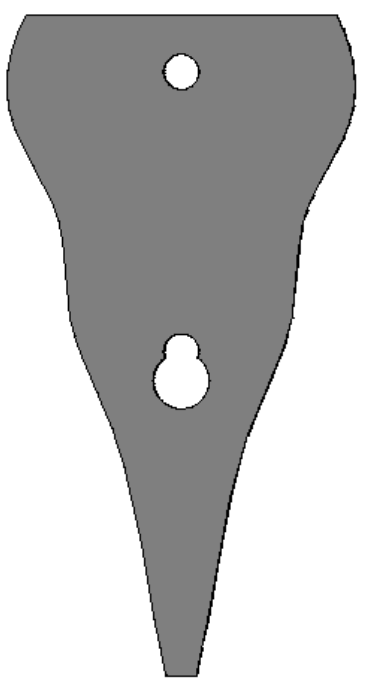

(c)

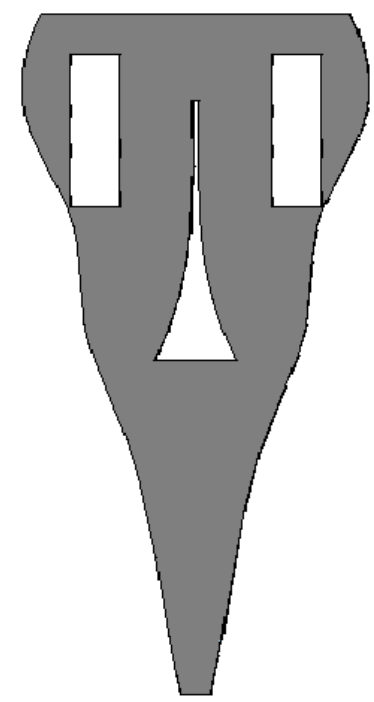

(g)

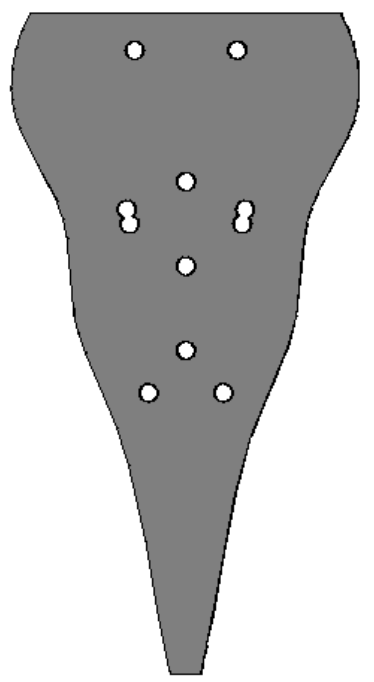

(d)

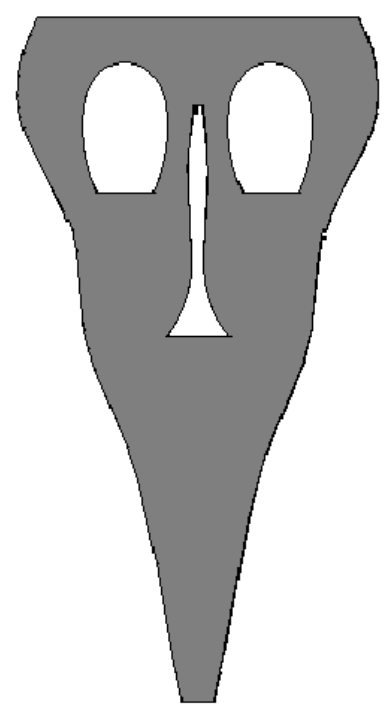

(h)

Fig. 10. Optimisation of the optimum "topology" designs, showing designs (a) to (h)

Looking at Fig. 10, it is difficult to assess, based on the shape alone, which design yields the highest power density. The design is determined by the average strain, power density, maximum von Mises stress, and structural volume across the piezoelectric element. Fig. 11 shows the power density and average strain for each design, while Fig. 12 shows the structural volume of the structure and von Mises stress. 


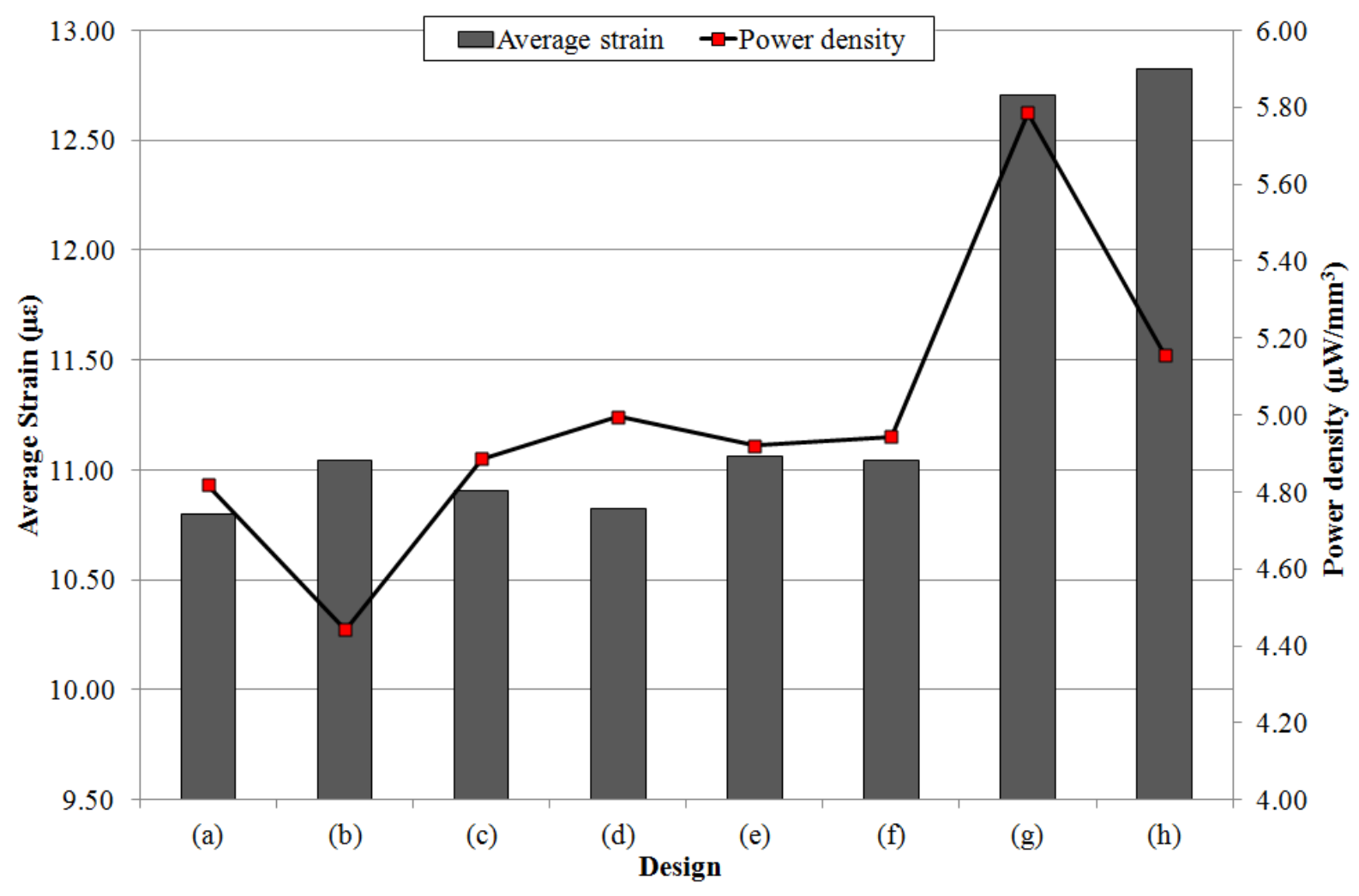

Fig. 11. Average strain and power density of the various optimum “topology” designs

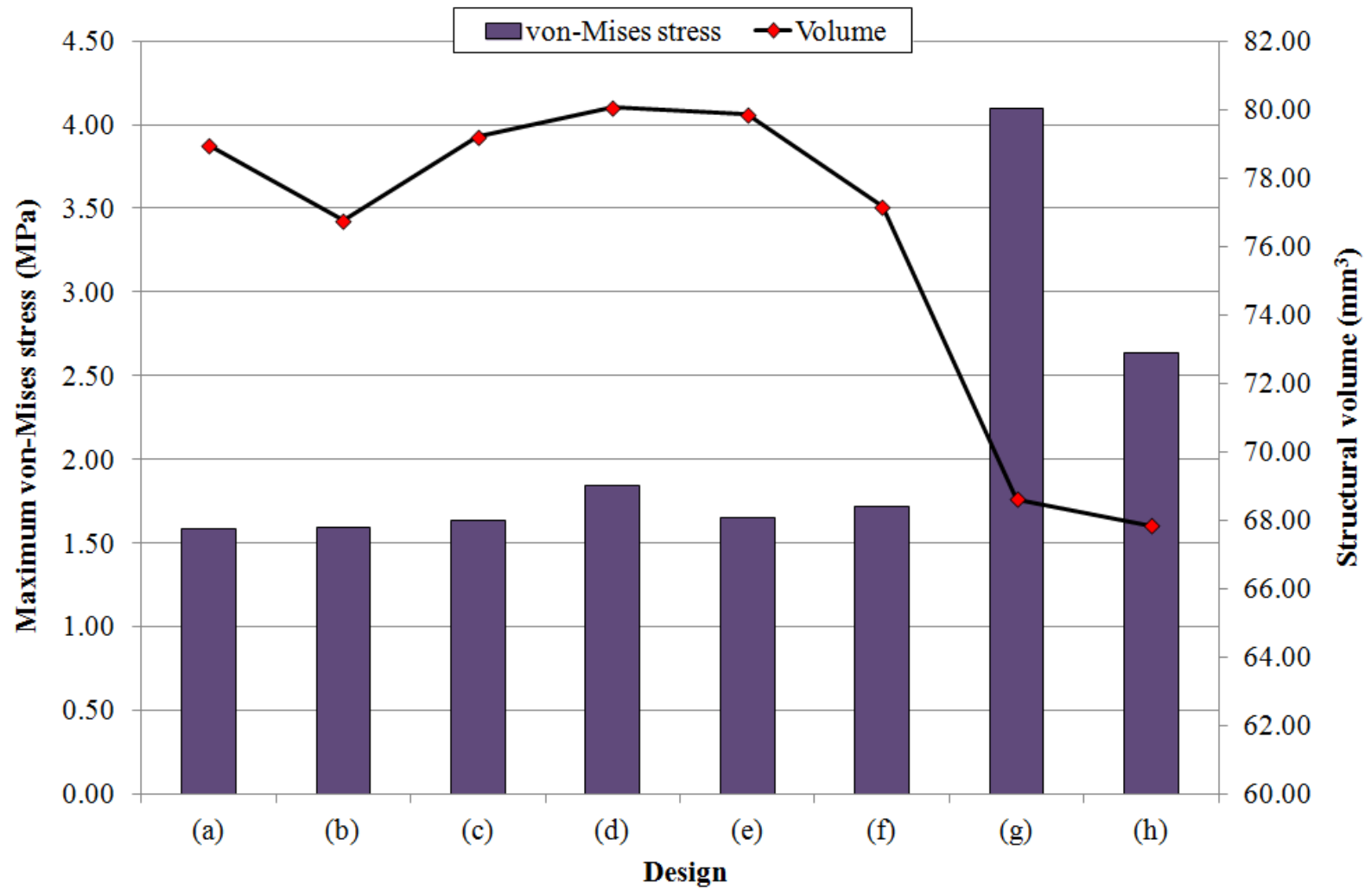

Fig. 12.von Mises stress and structural volume of the various optimum “topology” designs 
Figs. 11 and 12 show that designs (a) to (f) yield the low average strain, low power density, and low maximum von Mises stress. Conversely, designs (g) and (h) show the higher values. Among these designs, design (h) has the smallest structural volume, enabling the production of a cantilever beam at a lower cost. Although design (g) achieved the highest power density, it yielded a higher maximum von Mises stress than design (h). Design (g) would be another good design if power density or structural volume is one of the main criteria for a specific application. In this paper, optimising the overall performance is emphasized. . Therefore, the design (h) is the best "topology" design and is chosen as the design of the cantilever beam.

Fig. 13 shows the von Mises stress distribution for the optimum "topology" structure of design (h). The attributes of the optimised designs are listed in Table 7. The first-stage optimised structure shows a reduction in the maximum power output compared to the optimum "topology" structure. However, the power density per unit volume for the later design is increased by approximately 11\% compared with the first-stage optimised design. The structural volumes of the first-stage optimised and optimum "topology" designs of the cantilever beam (excluding the tip mass) are 81.83 and $67.84 \mathrm{~mm}^{3}$, respectively.

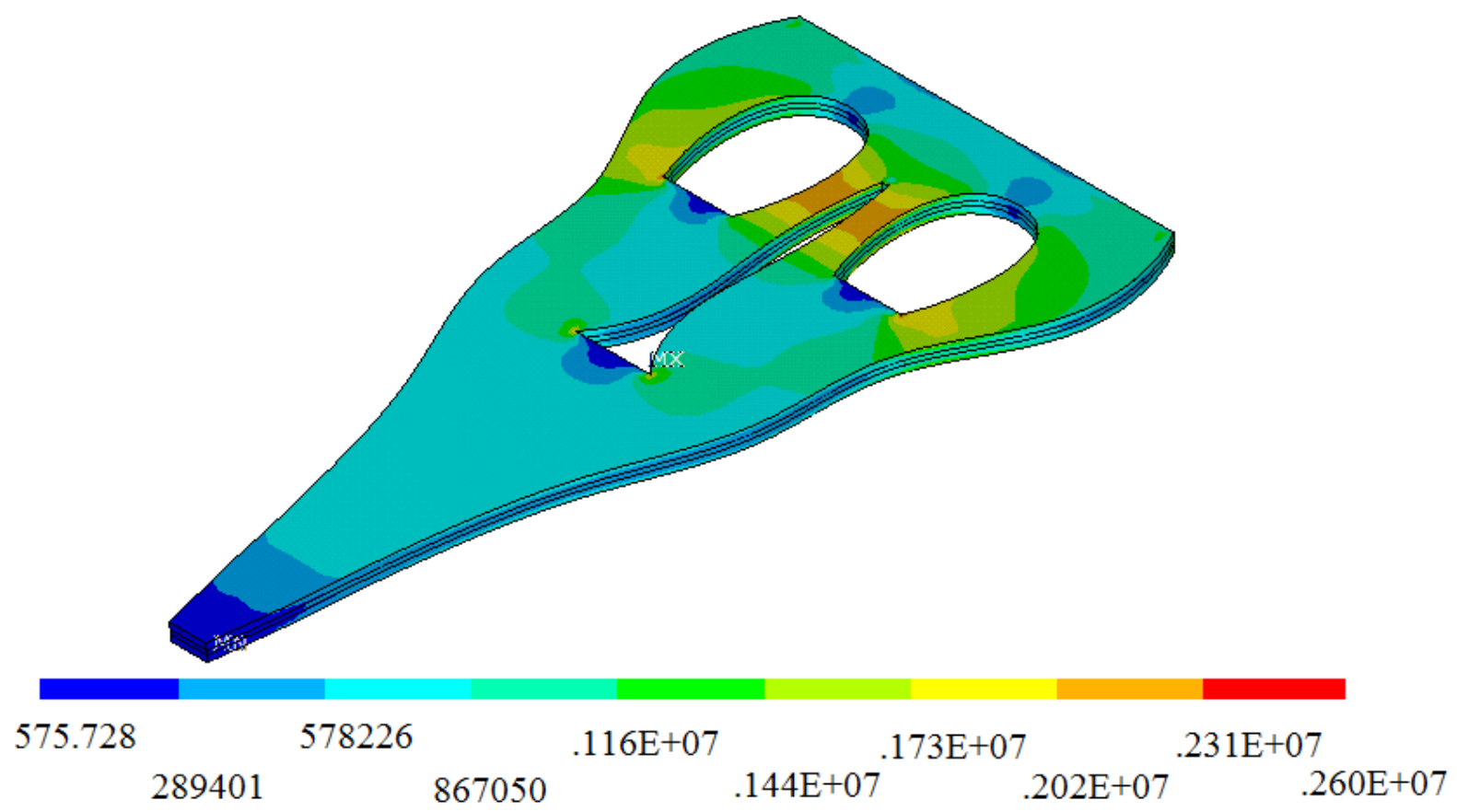

Fig. 13. Distribution of von Mises stress (Pa) in the optimum “topology” design (h)

Table 7 Design attributes of the first-stage optimised and optimum “topology” structures 


\begin{tabular}{lcc}
\hline & $\begin{array}{c}\text { First-stage } \\
\text { optimised design }\end{array}$ & $\begin{array}{c}\text { Optimum “topology” } \\
\text { design (h) }\end{array}$ \\
\hline Power density $\left(\mu \mathrm{W} / \mathrm{mm}^{3}\right)$ & 4.62 & 5.16 \\
Volume of piezoelectric material $\left(\mathrm{mm}^{3}\right)$ & 60.97 & 50.55 \\
Volume of shim material $\left(\mathrm{mm}^{3}\right)$ & 20.86 & 17.29 \\
Average element stress $(\mathrm{MPa})$ & 0.46 & 0.61 \\
Displacement at point $E(\mu \mathrm{m})$ & 12.50 & 18.30 \\
Capacitance $(\mathrm{nF})$ & 26.92 & 22.31 \\
Frequency $(\mathrm{Hz})$ & 93 & 78 \\
Power $(\mathrm{mW})$ & 0.282 & 0.261 \\
\hline
\end{tabular}

The higher power density of the optimum "topology" design than that of the first-stage optimised design reflects the fact that the volumes of the piezoelectric and shim materials are greatly reduced, yielding a $17.1 \%$ saving in material. The reduced structural volume is accompanied by a reduction in the capacitance of the structure. Although the average element stress of the optimum "topology" design is higher than that of the firststage optimised design, the power output is lower because the power equation of the cantilever beam does not rely solely on the average stress: it also depends on the frequency, damping ratio, resistance, and capacitance. The power output of the optimum "topology" structure is $0.261 \mathrm{~mW}$, which is $7.4 \%$ lower than that of the optimised design. Conversely, the average element stress of the optimum "topology" design is $32 \%$ higher than that of the optimised design. Similarly, the displacement at point $E$ is increased by $46.4 \%$ compared with the first-stage optimised design.

\section{Conclusions}

This paper presents an optimised design of a bimorph piezoelectric cantilever beam using the finite element method in conjunction with the multifactor optimisation of structures technique (MOST). A multi-objective and multi-disciplinary optimisation was performed for a 3D composite structural design in optimising the electrical energy performance (i.e., power density) and structural performance (i.e., volume, average element stress, displacement, and vibration frequency). The trade-off between the structural configuration and the power density of the cantilever beam represents a complication in terms of design optimisation.

First, a manual optimisation was performed and the results were compared with the results of an automatic optimisation. The results show a good agreement between the two methods in terms of seeking the optimum power density by utilising a holed structure (i.e., a hole opening within the design domain of the bimorph cantilever beam). Next, a two-stage optimisation was performed and a shape optimisation of the piezoelectric cantilever beam was presented as a first-stage design. Simulation results indicate that the optimised design can generate a power density of $4.62 \mu \mathrm{W} / \mathrm{mm}^{3}$ for a piezoelectric volume of $60.97 \mathrm{~mm}^{3}$. In the second stage, the 
power density was further increased to $5.16 \mu \mathrm{W} / \mathrm{mm}^{3}$ at a piezoelectric volume of $50.55 \mathrm{~mm}^{3}$. The results demonstrate the efficiency of the MOST technique.

In the Stage 2, the optimum "topology" design is also dependent on the weighting factors which are always related to the overall performance index (OPI) that control the optimisation process. Hence, the best "topology" is relevant to a specific weighting system. It is necessary to set the lower and upper bounds for each design variable providing a flexibility for the design variables to move within the design domain. Like any non-linear programming method, MOST may not necessarily achieve a global optimum or force every performance index to reach its optimum, especially when some objectives conflict with others that are concurrently being considered during the optimisation. However, the present results obtained for the bimorph cantilever beam demonstrate that the proposed method was successful in identifying the optimum design, resulting in improved performance in terms of power output and power density.

This paper concludes that the finite element analysis would be useful guides in maximising the power density of an energy-harvesting prototype device. For the future work, the theoretical analyses would be further validated through experiments. In addition, the MOST technique will be combined with a fully topology optimisation method to perform a single stage optimisation.

\section{References}

[1] J.M. Gilbert, F. Balouchi, Comparison of energy harvesting systems for wireless sensor networks, International Journal of Automation and Computing, 5(4) (2008) 334-347.

[2] S. Roundy, D. Steingart, L. Frechette, P. Wright, J. Rabaey. Power Source for Wireless Sensor Networks, Lecture Notes in Computer Science, Springer-Verlag, 2920 (2004) 1-17.

[3] S. Roundy, Energy Scavenging for wireless sensor nodes with a focus on Vibration to electricity conversion, PhD thesis, (2003) University of California Berkeley.

[4] L.M. Miller, N.C. Emley, P. Shafer, P.K. Wright, Strain Enhancement within Cantilevered, Piezoelectric MEMS Vibrational Energy Scavenging Devices, Advances in Science and Technology, Smart Materials \&Mico/Nanosystems, 54 (2008) 405-410.

[5] S. Roundy, On the effectiveness of vibration-based energy harvesting, Journal Intell. Material System Structure, 16 (2005) 809-823.

[6] L. Mateu, F. Moll, Optimum piezoelectric bending beam structures for energy harvesting using shoe inserts, J. Intell. Material System Structure, 16 (2005) 835-845.

[7] P. Simon, S.A. Yves, Electromechanical Performances of Different Shapes of Piezoelectric Energy Harvesters. International Workshop Smart Materials and Structures, 22 - 23 Oct (2009), Montreal, Canada

[8] L. Dhakar, H. Liu, F.E.H. Tay, C. Lee, A new energy harvester design for high power output at low frequencies. Sensors and Actuators, 199 (2013) 344-352

[9] S.X. Xu, T.S. Koko, Finite element analysis and design of actively controlled piezoelectric smart structures, Finite Element in Analysis and Design 40(3) (2004) 241-262. 
[10] J.S. Liu, L. Hollaway, Design optimisation of composite panel structures with stiffening ribs under multiple loading cases, Computers \& Structures, 78(4) (2000) 637-647.

[11] J.S. Liu, T.J. Lu, Multi-objective and multi-loading optimization of ultralightweight truss materials, International Journal of Solids and Structures, 41 (2004) 619-635.

[12] C.K. Thein, J.S. Liu, Effective structural sizing/shape optimisation through a reliability-related multifactor optimisation approach, Multidiscipline Modeling in Materials and Structures, 8(2) (2012) 159-177

[13] J.S. Liu, G. Thompson, The multi-factor design evaluation of antenna structures by parameters profile analysis, J. Engng Manufac. Proc Inst. Mech. Eng, 210(B5) (1996) 449-456.

[14] T. Ikeda, Fundamentals of piezoelectricity, Oxford science publications, (1996) Oxford.

[15] C.K. Thein, B.L. Ooi, J.S. Liu, J.M. Gilbert, Modelling and Optimisation of a Bimorph Piezoelectric Cantilever Beam in an Energy Harvesting Application, Journal of Engineering Science and Technology, 11(2) (2016) 212-227

[16] Q. Gallas, G. Wang, M. Papila, M. Sheplak, L. Cattafesta, Optimization of synthetic jet actuators, $41^{\text {st }}$ AIAA Aerospace Sciences Meeting and Exhibit, Reno, NV, USA, (2003) AIAA-2003-0635.

[17] Piezo System, Inc. CATALOG \#7C (2008), available online at http://www.piezo.com/catalog.html, [accessed on 31 July 2009].

[18] C.W. Bert, V. Birman, Effects of stress and electric field on the coefficients of piezoelectric materials: one-dimensional formulation, Mech. Res. Commun, 25(2) (1998) 165-9.

\section{Figures list}

Fig. 1.Details of the piezoelectric cantilever beam

Fig. 2. Schematic piezoelectric bender

Fig. 3. Power density of different designs

Fig. 4. Power density obtained for different hole locationsin a rectangular bimorph cantilever beam

Fig. 5. Design variables of the initial design in Stage 1

Fig. 6. Optimisation convergence history of the structural volume and power

Fig. 7. Distribution of von Mises stress for the initial design (left) and for the optimised design (right) (Pa)

Fig. 8.Design domain and shape constraints of the optimised design

Fig. 9. Initial "topology” hole openings, showing designs (a) to (h)

Fig. 10. Optimisationof the optimum "topology" designs, showing designs (a) to (h)

Fig. 11. Average strain and power density of the various optimum "topology" designs 
Fig. 12. von Mises stress and structural volume of the various optimum "topology” designs

Fig. 13. Distribution of von Mises stress in the optimum "topology" design (h) (Pa) 